\title{
On Estimating the Topology of an Adversarial Wireless Network
}

\author{
Andrew W. Eckford and Scott E. T. Hadley \\ Department of Computer Science and Engineering, York University \\ 4700 Keele Street, Toronto, Ontario, Canada M3J 1P3 \\ Emails: aeckford@yorku.ca, cs262009@cse.yorku.ca
}

\begin{abstract}
Owing to recent interest in sensor networks for military and security applications, studies of the security vulnerabilities of these networks are becoming increasingly important. In the present paper, the problem of estimating the topology of an adversarial sensor network is considered. The adversarial network is assumed to employ strong encryption, so that its transmitted packets are assumed to be unreadable by the observer. Thus, the algorithms are required to make use of the time correlations in channel uses by the adversarial network. Assuming the use of the MACA protocol, our algorithms are capable of estimating both the routes used by nodes in the adversarial sensor network, and as the identities of the nodes that are within each other's neighborhood (i.e., within radio range of each other), so that an attack could be designed for maximum effect. Results are presented which show that route estimation can be accomplished quickly using our algorithm, and that neighborhood estimation can be accomplished in a reasonable amount of time.
\end{abstract}

\section{INTRODUCTION}

Wireless sensor networks [1], which allow the monitoring of a physical phenomenon over a wide geographic area, have attracted a great deal of recent attention. These networks have demonstrated their effectiveness in applications as diverse as wildlife tracking [2] and structural monitoring [3].

The data-gathering ability of these networks make them a natural fit for military and intelligence-gathering applications, either offensive (in the sense of espionage) or defensive (in the sense of security). As a result, we can consider the possibility of an adversarial wireless network, which is acting against one's own interests, and whose activities would be desirable to disrupt. For example, an adversary could deploy a sensor network to monitor the positions and activities of friendly forces. For an adversary with reasonable technical skills, this would be a relatively cheap and highly effective strategy for tactical intelligence gathering, which could be accomplished using offthe-shelf hardware. The sensor nodes could be sufficiently small and well-hidden that, unless specific countermeasures were taken, it is unlikely that friendly forces would discover the sensor network until they were well inside the "digital minefield" (i.e., the effective range of the adversarial network).

Due to the topology and node distribution of a typical sensor network, the sensor nodes typically do not have a (strong) direct link to the data sink. Thus, sensor nodes generally need help (e.g., routing or relaying) from neighboring nodes in order for its transmission to arrive successfully at the data sink. As such, sensor networks are vulnerable to homing attacks, in which key nodes (such as relays) are identified and destroyed [5]. Some researchers working on this problem focus on network intrusion, in which a node under friendly control becomes a member of the adversarial network, and can then observe the sources, routes, destinations, and contents of packets [7]. However, this strategy is easily defeated by cryptography.

On the other hand, since wireless sensor networks necessarily emit RF energy, traffic analysis can be performed on these free-space RF transmissions to determine the identities of relays (e.g., by observing the correlations in time between two nodes' transmissions). This approach was previously taken in [8], which was mostly concerned with identifying a base station (which we refer to as a data sink). Other approaches can be taken, such as observing the total data rate through each node, with the largest data rate most likely corresponding to the data sink [9]. Although these approaches are more robust than network intrusion, they have certain limitations. In particular, although they might identify data paths, they do not give any idea as to how the network might reconfigure itself if attacked.

In this paper, we consider wireless networks that implement the Multiple Access with Collision Avoidance (MACA) protocol [10], a medium access protocol in which the neighborhood of a node is "cleared" by request-to-send (RTS) and clearto-send (CTS) messages, thereby avoiding data collisions. This protocol is useful for sensor networks, and has been extended in several ways that are specifically applicable to the ad-hoc and sensor networking problem [11], [12]. An interesting feature of MACA is that a data path not only affects the behavior nodes along the path, but also all nodes within radio range of the path. Exploiting this fact, our main contribution in this paper is a set of algorithms that not only estimate data paths in an adversarial wireless network using MACA, but also estimate which nodes are in range of which other nodes. As a result, an attacker can predict how the network might reconfigure itself if attacked, and can thus design an attack for maximum effect. Our results show that estimating the routes can be accomplished quickly using our algorithm, while estimating the neighborhoods can be accomplished in a reasonable amount of time. As a result, the use of strong encryption is not sufficient to prevent an observer from gathering important information about a sensor network. 
The remainder of the paper is organized as follows. In Section II, we introduce our system models and all relevant assumptions concerning our setup. In Section III, we state our algorithms for estimating data paths and for estimating radio neighborhoods under two different modeling assumptions. In Section IV, we present the results of our algorithms, and discuss how our algorithms could be modified to cope with different modeling assumptions.

\section{MODEL}

The network to be observed, known as the adversarial network, is assumed to be a wireless network. The goal of each node in the network is to communicate its information to some data sink. To accomplish this task, the nodes in the network possess a routing table, in which there are no isolated nodes, and in which there is a unique route for a given node's packets to reach the data sink. Packets are passed as datagrams (via, e.g., UDP), so there is no end-to-end acknowledgment of data transmission.

For simplicity, throughout the remainder of the paper we will assume that time is slotted, so that all events occur at integer time units $t \in\{1,2, \ldots\}$. At each time instant $t$, there exists a probability $p$ that any node will have information to transmit to the sink, independent of any other node; furthermore, the node may be required to route neighboring nodes' data packets to the data sink. For nodes that wish to send information, the adversarial network employs MACA for medium access control, which functions as follows to transmit information from node A to node B [10]:

1) (Time $t$ ): Node A transmits a request-to-send (RTS) message to node $\mathrm{B}$.

2) Node $\mathrm{B}$ waits $k \in\{1,2, \ldots\}$ units of time until it is ready to receive node A's information (where $k=1$ if $\mathrm{B}$ is ready to receive the data immediately).

3) (Time $t+k$ ): Node B transmits a clear-to-send (CTS) message to node A.

4) (Time $t+k+1$ ): Node A sends a DATA message (containing the data to be sent) to node B.

5) (Time $t+k+2$ ): Node $\mathrm{B}$ sends an acknowledgment (ACK) message to node A.

Between the transmission of CTS and ACK, all nodes in radio range of $\mathrm{B}$ (other than $\mathrm{A}$ ) must remain silent, so as to avoid interfering with $\mathrm{A}$. Note that all possibly interfering nodes observe both the CTS and ACK, since they are in radio range. The $k$ time unit wait in step 2 may arise, for example, if $\mathrm{B}$ is in range of a different node that has transmitted a CTS. Additionally, for simplicity, we assume that the messages are always transmitted successfully.

There are two graphs representing the adversarial network, with vertices representing nodes in the network, and edges representing connections in the network. First, there is the routing graph $\mathcal{R}$, which is a directed graph in which an edge exists from vertex A to vertex B if and only if B is on A's path to the data sink (or vice versa). Second, there is the neighborhood graph $\mathcal{N}$, which is an undirected graph in which

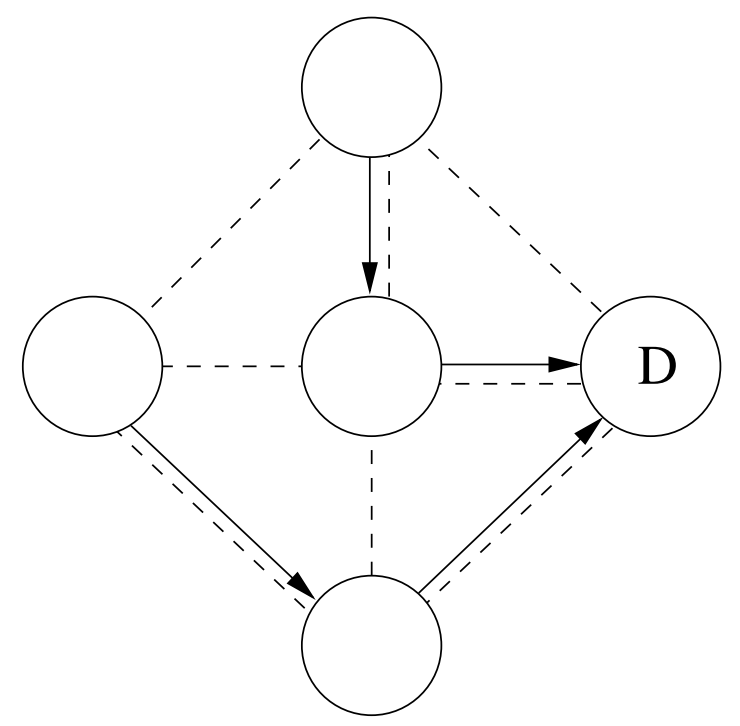

Fig. 1. Routing graph $\mathcal{R}$ and neighborhood graph $\mathcal{N}$ for an example network, where $\mathcal{R}$ is represented by the solid lines, and $\mathcal{N}$ is represented by the dashed lines. The data sink is at node D.

an edge exists between vertices A and B if they are in radio range of each other. An example is depicted in Figure 1.

The adversarial network is monitored by an observer, whose task is to estimate both $\mathcal{R}$ and $\mathcal{N}$. It is assumed that the adversarial network is employing a strong encryption scheme, so that neither the data nor the packet headers sent in the adversarial network are available to the observer. However, it is assumed that the observer can identify exactly when each packet is transmitted, and by whom (for example, if the nodes are distributed randomly in space, and the channel is static, each node is likely to have a unique signal strength from the observer's perspective, which can be used to correlate transmissions with nodes). Furthermore, we adopt two possible assumptions with respect to the packets:

1) (Assumption RCAD) The observer can distinguish each packet: RTS, CTS, ACK, and DATA. However, the observer cannot read the packets (and thus cannot distinguish the intended recipient of the packets). This assumption is valid if RTS, CTS, and ACK have different physical characteristics, such as different lengths.

2) (Assumption XD) The observer can distinguish the control packets (RTS, CTS, and ACK) from the DATA packets, but cannot distinguish among the control packets (i.e., the observer can't tell which control packet is RTS, CTS, or ACK). Furthermore, the observer cannot read any packet. This assumption is valid in general since the DATA packet is likely to be much longer in duration than any control packet.

For convenience, we assume that the observer is aware of the number of nodes in the network in advance, although our algorithms can be reworked in order to relax this assumption.

Let $\mathcal{I}=\{1,2, \ldots,|\mathcal{I}|\}$ represent an index set corresponding 
to the nodes in the adversarial network. Under each observer assumption, we can map each possible message to a digit: for instance, under assumption $\mathrm{XD}$, we can use 1 to represent control packets and 2 to represent DATA packets; under assumption RCAD, we can use 1, 2, 3, and 4 to represent RTS, CTS, ACK, and DATA packets, respectively; and under either assumption, we can use 0 if no message was transmitted. Given a modeling assumption, let $\mathcal{M}$ represent this set of possible messages. Furthermore, for $i \in \mathcal{I}$, let $y_{t, i}$ represent the value corresponding to the message transmitted by node $i$ at time $t$. Then at each time $t \in\{1,2, \ldots\}$, the observer obtains a vector $\mathbf{y}_{t}=\left[y_{t, 1}, y_{t, 2}, \ldots, y_{t,|\mathcal{I}|}\right]$, with elements taken from $\mathcal{M}$, corresponding to the radio activity of the adversarial network at time $t$. In this paper, we do not consider the case where the observations $\mathbf{y}_{t}$ contain errors.

\section{ESTIMATION ALGORITHMS}

\section{A. Overview and Definitions}

In this section, we give estimation algorithms that are performed by the observer to estimate $\mathcal{R}$ and $\mathcal{N}$. The algorithms are separated into edge detection algorithms, to estimate $\mathcal{R}$; and space detection algorithms, to estimate $\mathcal{N}$. In each case, we give algorithms for assumptions RCAD and XD.

In general, we take a rule-based approach to this problem; that is, we identify configurations of the set of vectors $\mathbf{y}_{t}$ that are only possible if particular edges are present (or absent) in $\mathcal{R}$ or $\mathcal{D}$. For example, in the edge detection problem, observing a CTS from node A, immediately followed by a DATA packet from node $\mathrm{B}$, followed by an ACK from node A, would indicate that there is an edge between B and A in $\mathcal{R}$. Similarly, for the space detection problem, one of our approaches is to determine which nodes transmit simultaneously with a DATA packet, as these nodes cannot be in the neighborhood of the node transmitting the DATA packet.

We give some definitions that will be useful in this section. Recall that $\mathcal{I}$ is the index set of the nodes, and $\mathcal{M}$ is the set of radio messages. Let

$$
I: \mathcal{M}^{|\mathcal{I}|} \times \mathcal{M} \rightarrow\{0,1\}^{|\mathcal{I}|}
$$

represent a vector indicator function, where if $\mathbf{u}_{t}=I\left(\mathbf{y}_{t}, x\right)$ for an observation $\mathbf{y}_{t}$ and a message $x \in \mathcal{M}$, then

$$
u_{t, i}= \begin{cases}1, & y_{t, i}=x \\ 0, & y_{t, i} \neq x\end{cases}
$$

In other words, $\mathbf{u}_{t}$ is 1 wherever the message $x$ is found in $\mathbf{y}_{t}$, and 0 everywhere else. Furthermore, for two vectors of the same length $\mathbf{a}$ and $\mathbf{b}$, define the $\cdot$ operation $\mathbf{a} \cdot \mathbf{b}$ as forming the vector by multiplying the individual vector elements (i.e., $\left.\mathbf{a} \cdot \mathbf{b}=\left[a_{1} b_{1}, a_{2} b_{2}, a_{3} b_{3}, \ldots\right]\right)$. For a matrix $\mathbf{M}$, the $i$ th row and $j$ th column of the matrix will be written $\mathbf{M}_{i}$ and $\mathbf{M}_{j}^{\prime}$, respectively; while the $(i, j)$ th element will be written $M_{i, j}$.

\section{B. Edge detection}

1) Assumption RCAD: Briefly speaking, from our description of the MACA protocol, we see that CTS, DATA, and ACK are all transmitted in consecutive slots. Thus, the key to our algorithm is to identify CTS-ACK cycles; any DATA packets that are transmitted in these cycles comes from a node connected by a possible edge in $\mathcal{R}$. There is ambiguity if more than one CTS-ACK cycle occurs at once. However, it is unlikely that this ambiguity will persist for many uses of the same link. Once there is only one possible pair of nodes that are communicating over a given link, the corresponding edge in $\mathcal{R}$ is declared to be present.

More formally, the algorithm proceeds as follows. For clarity, we will refer to messages in $\mathcal{M}$ by their names (RTS, CTS, ACK, and DATA) rather than by number.

1) (Initialization step.) Let $t=1$. Let $\mathbf{P}$ be a $|\mathcal{I}| \times|\mathcal{I}|$ matrix where every element is 1 . ( $\mathbf{P}$ is the matrix of possible edges: if $P_{i, j}=1$, then it is possible for an edge to exist from $i$ to $j$ in $\mathcal{R}$; if $P_{i, j}=0$, then such an edge is ruled out by previous evidence.)

2) Obtain $\mathbf{y}_{t}$. If $t<3$, go to 5 . (We need at least three vectors $\mathbf{y}_{t}$ to start the estimation algorithm.)

3) Let $\mathbf{u}_{t}=I\left(\mathbf{y}_{t}, A C K\right) \cdot I\left(\mathbf{y}_{t-2}, C T S\right)$. (Thus, $\mathbf{u}_{t}$ is 1 wherever a CTS-ACK cycle has taken place.)

4) Let $\mathbf{d}_{t}=I\left(\mathbf{y}_{t-1}, D A T A\right)$. For each $i \in\{1,2, \ldots,|\mathcal{I}|\}$, if $d_{t, i}=1$, then let $\mathbf{P}_{i}:=\mathbf{P}_{i} \cdot \mathbf{u}_{t}$. (For a DATAsending node $i$, possible edges only exist to nodes in the current CTS-ACK cycle; we also rule out nodes that were previously ruled out by $\mathbf{P}_{i}$.)

5) Let $t:=t+1$ and go to 2 .

The results of the algorithm are contained in $\mathbf{P}$. Note that an edge has been positively identified for each $\mathbf{P}_{i}$ that contains a single 1 ; if the single 1 is in the $j$ th position, then the edge exists from vertex $i$ to vertex $j$. Note that the data sink never transmits a DATA message - it only transmits CTS and ACK messages to receive data - which is a possible way to quickly identify the data sink.

Under the modeling assumptions we have made, a positive detection of an edge in $\mathcal{R}$ is error-free, as there are no remaining ambiguities in the identities of the sender and receiver.

2) Assumption $X D$ : This algorithm proceeds similarly to the RCAD case. However, since CTS and ACK messages cannot be distinguished, the algorithm now looks for a sequence corresponding to: control message, followed by DATA, followed by control message. This may introduce additional ambiguity, since other control messages might masquerade as CTS and ACK, but again, it is unlikely that this ambiguity will persist for many uses of the same link. In particular, if the two types of messages under the $\mathrm{XD}$ assumption are referred to as CTRL and DATA, we only need to change step 3 of the algorithm to the following:

$$
\text { 3) Let } \mathbf{u}_{t}=I\left(\mathbf{y}_{t}, C T R L\right) \cdot I\left(\mathbf{y}_{t-2}, C T R L\right) \text {. }
$$

This is sufficient to identify all CTS-ACK cycles, although it will misidentify other control message traffic as a CTS-ACK cycle as well, hence increasing the ambiguity and requiring a longer period of observation to identify $\mathcal{R}$. However, at termination, $\mathcal{R}$ is again identified without errors. 


\section{Space detection}

Throughout this section, for each node $i \in\{1,2, \ldots,|\mathcal{I}|\}$, define the function $R: \mathcal{I} \rightarrow \mathcal{I}$ so that, if $j=R(i)$, then $j$ is the first node traversed in the path from $i$ to the data sink (or $j$ is the data sink if $i$ is adjacent to it). The function $R(i)$ is easily obtained from $\mathbf{P}$, the result of the edge detection algorithm.

1) Assumption RCAD: The space detection problem, in which $\mathcal{N}$ is estimated, is divided into two sub-problems: we estimate the negative space, consisting of all nodes known to be outside the neighborhood of a node (i.e., unconnected in $\mathcal{N}$ ); and we also estimate the positive space, consisting of all nodes known to be within the neighborhood of a node (i.e., connected in $\mathcal{N}$ ).

We give the negative space algorithm first, which relies on the fact that, if a node is sending DATA at time $t$, any other node also transmitting any message at time $t$ cannot be within radio range of the DATA message's destination (because that neighborhood is cleared of radio traffic). The algorithm proceeds as follows.

1) (Initialization step.) Run the edge detection algorithm to obtain $\mathbf{P}$ (i.e., $R(i)$ is assumed known). Let $\mathbf{N}$ be a $|\mathcal{I}| \times|\mathcal{I}|$ all-one matrix (and similarly to $\mathbf{P}$ in step 1 of the edge detection algorithm, $\mathbf{N}$ stores our intermediate results).

2) Obtain $\mathbf{y}_{t}$. Let $\mathbf{n}_{t}=I\left(\mathbf{y}_{t}, C T S\right)+I\left(\mathbf{y}_{t}, R T S\right)+$ $I\left(\mathbf{y}_{t}, A C K\right)+I\left(\mathbf{y}_{t}, D A T A\right)$ (Note that the elements of $\mathbf{n}_{t}$ only take values in $\{0,1\}$; further, note that $n_{t, i}$ is 1 if and only if node $i$ is sending any message, and zero otherwise).

3) Let $\mathbf{d}_{t}=I\left(\mathbf{y}_{t}, D A T A\right)$. For each $i \in\{1,2, \ldots,|\mathcal{I}|\}$, if $d_{t, i}=1$, then $\mathbf{N}_{R(i)}:=\left(1-\mathbf{n}_{t}\right) \cdot \mathbf{N}_{R(i)}$, and $\mathbf{N}_{R(i)}^{\prime}:=$ $\left(1-\mathbf{n}_{t}\right)^{\prime} \cdot \mathbf{N}_{R(i)}^{\prime}$. (Nodes transmitting at the same time as a DATA-sending node are outside of its space, and vice versa.) Let $\mathbf{N}_{R(i), R(i)}:=1$ (a node is clearly in its own neighborhood; this was set to zero in the previous statement).

4) Let $t:=t+1$, and go to 2 .

The results of the algorithm are contained in the matrix $\mathbf{N}$. If $N_{i, j}=0$, then nodes $i$ and $j$ are disconnected in the graph $\mathcal{N}$ (however, we draw no conclusion if $N_{i, j}=1$ ). Again, this algorithm provides results that are free of ambiguity, so any zeros in $\mathbf{N}$ indicate edges that are certainly absent in $\mathcal{N}$.

Given enough time, it is likely that the negative space algorithm would find the entire structure of $\mathcal{N}$. However, a quicker determination of the neighborhoods can be made by directly detecting the positive space. To do this, we consider those cases where a node $i$ sends an RTS message to node $j$, but the responding CTS from $j$ is not transmitted immediately. In these cases, one reason for this delay to occur is because $j$ is blocked from transmitting by another CTS in its neighborhood (the other reason, which must be dealt with by the algorithm, is that $j$ is in the midst of its own RTS-DATA cycle, which must be completed before it can respond with CTS). Thus, we can identify nodes that are neighbors of $j$ by counting those nodes that send a CTS that seems to block a CTS from $j$. However, unlike the preceding algorithms, it is not possible to identify blocking CTS messages with certainty, so this algorithm introduces some possibility of error.

The algorithm proceeds as follows, and is intended to be run simultaneously with the negative space algorithm.

1) As in the negative space algorithm, run the edge detection algorithm to obtain $\mathbf{P}$ (i.e., $R(i)$ is assumed known). Let $\mathbf{M}$ and $\mathbf{B}$ be $|\mathcal{I}| \times|\mathcal{I}|$ all-zero matrices to store intermediate results. Let $\mathbf{s}_{t}$ be an all-zero vector of length $|\mathcal{I}|$ (this vector keeps track of the nodes that are in the midst of RTS-DATA cycles).

2) Obtain $\mathbf{y}_{t}$. Let $\mathbf{d}_{t}=I\left(\mathbf{y}_{t}, D A T A\right), \mathbf{r}_{t}=I\left(\mathbf{y}_{t}, R T S\right)$, and $\mathbf{c}_{t}=I\left(\mathbf{y}_{t}, C T S\right)$.

3) Let $\mathbf{s}_{t}:=\mathbf{s}_{t}+\mathbf{r}_{t}-\mathbf{d}_{t}$. (Note that the elements of $\mathbf{s}_{t}$ only take values in $\{0,1\}$; further, note that $s_{t, i}=1$ if node $i$ is in an RTS-DATA cycle, and zero otherwise.)

4) For each $i \in\{1,2, \ldots,|\mathcal{I}|\}$, if $r_{t, i}=1$, then $\mathbf{M}_{i}:=$ $[0,0, \ldots, 0]$ (so the $i$ th row of $\mathbf{M}$ will count the number of CTS messages in the network in the interval between $i$ sending RTS and DATA; this initializes the count starting with the RTS from $i$ ).

5) For each $i, j \in\{1,2, \ldots,|\mathcal{I}|\}$, if $s_{t, R(i)}=0$ and $c_{t, j}=$ 1 , then $M_{i, j}:=M_{i, j}+1$ (i.e., for each CTS message in the network, increment the waiting counts for all nodes by 1 , only for those nodes $i$ where $R(i)$ is not in the midst of an RTS-DATA cycle).

6) For each $i \in\{1,2, \ldots,|\mathcal{I}|\}$, if $d_{t, i}=1$, then $\mathbf{B}_{i}:=$ $\mathbf{B}_{i}+\mathbf{M}_{i}$ (so $\mathbf{B}_{i}$ contains the count of time units that node $i$ has spent waiting for each other node).

7) Let $t:=t+1$, and go to 2 .

To obtain the positive space, we inspect B. For $i, j \in$ $\{1,2, \ldots,|\mathcal{I}|\}$, if $B_{i, j}>0$, then node $i$ appeared to wait for at least one CTS from node $j$. Thus, we conclude that there exists an edge between $R(i)$ and $j$ in $\mathcal{N}$ if $B_{i, j}>0$ and $N_{R(i), j} \neq 0$ (because the slower negative space algorithm is running simultaneously, which provides completely reliable results on the negative space).

2) Assumption $X D$ : In the negative space detection algorithm, our only reference to RTS, CTS, and ACK control messages (which are indistinguishable under assumption XD) was in step 2 . We can replace step 2 with

2) Obtain $\mathbf{y}_{t}$. Let $\mathbf{n}_{t}=I\left(\mathbf{y}_{t}, C T R L\right)+I\left(\mathbf{y}_{t}, D A T A\right)$.

Since this is the only change, it is easy to see that this algorithm is identical to the algorithm under assumption RCAD.

To obtain the positive space algorithm, we make the following changes. First, in step 2, we let $\mathbf{r}_{t}=I\left(\mathbf{y}_{t}, C T R L\right)$; since an RTS message must precede a DATA message, the extra resets prompted by spurious control messages have no effect. Furthermore, we let $\mathbf{c}_{t}=I\left(\mathbf{y}_{t+1}, D A T A\right)$, since DATA messages must follow CTS messages; this introduces a delay of one time unit to make the algorithm causal. We omit step 3 , with its calculation of $\mathbf{s}_{t}$; however, it is possible to identify RTS-DATA cycles by examining when DATA messages are transmitted, and correlating them with the most recent CTRL 


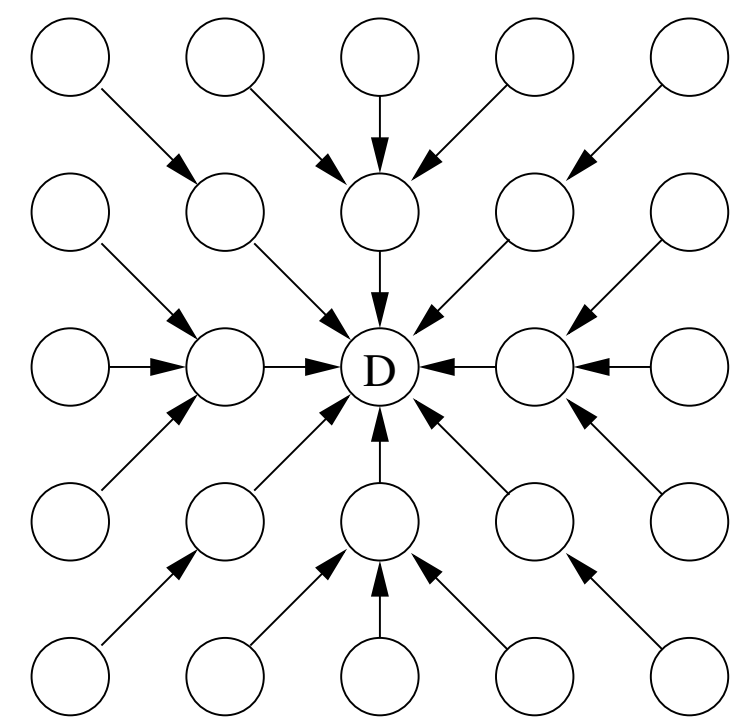

Fig. 2. Routing graph $\mathcal{R}$ for the 5-by-5 network used in simulation. Adjacent nodes (horizontal, vertical, and diagonal) on the graph are within radio range of each other. The data sink is at node $\mathrm{D}$.

message originating from the same node (which must have been an RTS). In step 5, because $\mathbf{s}_{t}$ is no longer available, we initially assume $s_{t, R(i)}=0$ and adjust $M_{i, j}$ when the RTSDATA cycles are unambiguous.

\section{RESUlts AND Discussion}

\section{A. Simulation results}

We ran our algorithms on networks with nodes arranged on a grid, routes determined by a "star" topology, and neighborhoods governed by adjacency in the grid. An example network is depicted in Figure 2 for a 5-by-5 grid, though analogous 7by-7 and 9-by-9 grids were also used. In each case, we assume that the nodes generate data in a time slot with probability $p=0.00625$ for the 5-by-5 grid, $p=0.00446$ for the 7-by7 grid, and 0.00312 for the 9-by-9 grid (these values were chosen as realistic values to prevent the neighborhood of the data sink from becoming saturated with traffic). Once data is generated, it is routed to the data sink at the earliest possible opportunity. The algorithms observe the adversarial network for $10^{5}$ time units $t$. Under a conservative assumption of 10 slots per second, this means an observation time of $10^{4}$ seconds, or around 2 hours, 47 minutes.

Results for the edge detection algorithm, the negative space detection algorithm, and the positive space detection algorithm are given in Figures 3, 4, and 5, respectively. For the edge detection algorithm, we see that the algorithm very rapidly succeeds in completely estimating $\mathcal{R}$ (we only include the 9by- 9 grid on this plot). We mentioned that the positive space detection algorithm generates errors, but we do not plot the error results since the number of misidentified edges was very low - fewer than ten in each simulation given.

The two space detection algorithms are much slower to estimate $\mathcal{N}$. It is not especially surprising that these algorithms

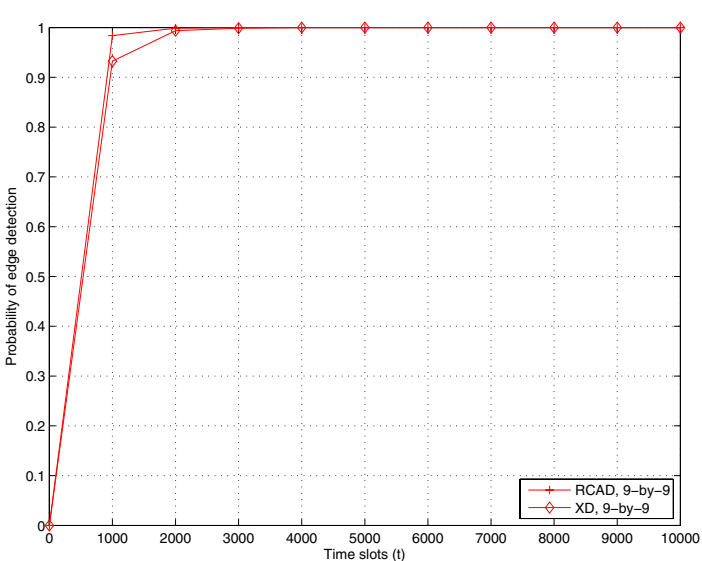

Fig. 3. Results for the edge detection algorithm.

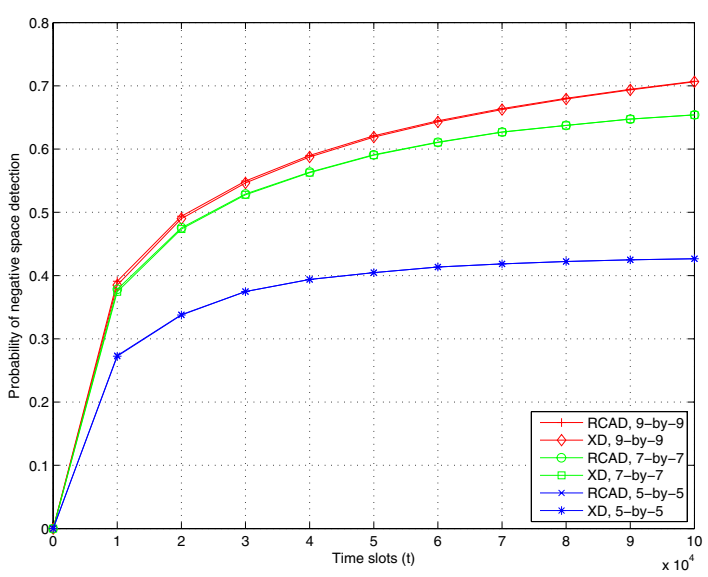

Fig. 4. Results for the negative space detection algorithm.

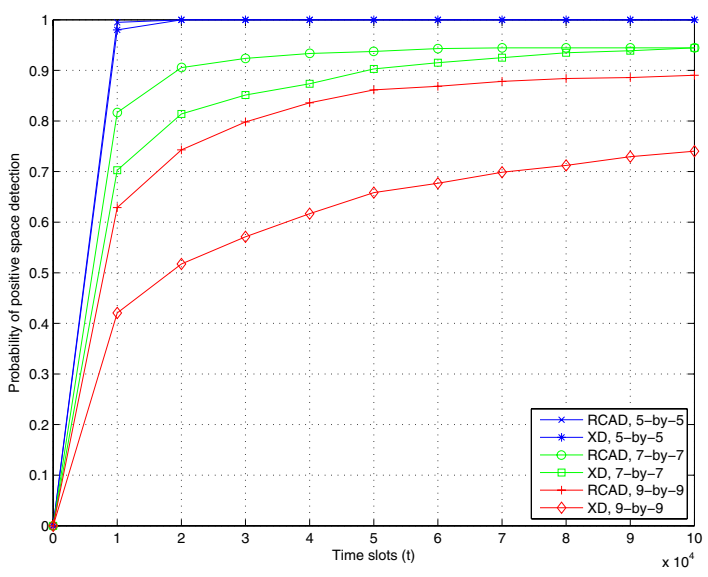

Fig. 5. Results for the positive space detection algorithm. 
run more slowly, since they rely largely on coincidental events to gather information. However, the algorithms nonetheless succeed in identifying significant portions of the graph $\mathcal{N}$ in a relatively short period of time; indeed, their performance is remarkable considering the small values of $p$ in use in the network. Furthermore, we note that the algorithms under assumption XD are not much worse than under assumption RCAD, indicating that very little information is required about the adversarial network's transmissions to do a good job of detection.

\section{B. Discussion}

In some cases, such as for positive space detection and edge detection, we see that the speed of convergence decreases for larger networks. This is potentially due to our model of data gathering: we assume that, in each time slot, a single observation is made of the entire radio traffic in the area. However, for an adversarial network distributed over a large area, this would not reflect the way data would be gathered in practice. Indeed, one might deploy a friendly sensor network to gather information about the adversarial sensor network, in which case multiple local measurements would be taken. These additional measurements would add fidelity to the detection problem, since (for example) the radio ranges of the friendly network would be comparable to those of the adversarial network, so the problem would be naturally partitioned into regions of the graph. As a result of this observation, work is proceeding on distributed versions of these algorithms, from which we anticipate even better performance.

Our algorithms can be straightforwardly adapted to alternative scenarios that we have not considered. As one example, in our space detection algorithms we took advantage of the fact that data always takes a single route to the data sink. In a network where this was not true, it would be possible to inspect the control message traffic to guess the sourcedestination pair for any given packet. As another example, we have assumed that messages passed within the adversarial graph are always error-free. To relax this assumption, we would have to allow for behavior in the adversarial graph that did not exactly conform to the MACA protocol. This could be handled by rejecting any non-conforming observations, at the cost of delaying the estimation.

Taking the problem of non-conforming behavior further, it is possible to imagine that an adversarial network would deliberately take action to frustrate the estimation problem, as pointed out in [8]. However, we conjecture that any such action would degrade the performance of the adversarial network. For example, an adversarial network could abandon MACA and use a TDMA scenario, in which each node always transmitted in its alloted slot whether or not it had useful information to send. In this case, estimating the routes to the data sink would be impossible. However, the adversarial network would be wasting a significant amount of energy to transmit useless information. Investigating the tradeoff between detectability and efficiency is a focus of future work.
Aside from the topics we have mentioned, our future work in this area will also adapt our algorithms to continuous time (non-slotted) scenarios, and also investigate similar detection algorithms for other sensor network MAC protocols.

\section{CONCLUSION}

In this paper, we have presented algorithms for detecting the topology of an adversarial sensor network using MACA. Our results indicate that, even if the adversarial network is employing strong encryption so as to prevent an outsider from deciphering its packets, it is still possible to quickly obtain the routing table. Furthermore, given some extra time, it is possible to determine which nodes are within the radio ranges of each other. These results indicate that encryption alone is insufficient to prevent an adversary from gathering useful intelligence about a network.

\section{REFERENCES}

[1] C.-Y. Chong and S. P. Kumar, "Sensor networks: Evolution, opportunities, and challenges," Proceedings of the IEEE, vol. 91, no. 8, pp. 1247-1256, Aug. 2003.

[2] P. Zhang, C. M. Sadler, S. Lyon, and M. Martonosi, "Hardware design experiences in ZebraNet," Proc. 2nd International Conference on Embedded Network Sensor Systems, Baltimore, MD, USA, pp. 227-238, 2004.

[3] N. Xu et al., "A wireless sensor network for structural monitoring," Proc. 2nd International Conference on Embedded Network Sensor Systems, Baltimore, MD, USA, pp. 13-24, 2004.

[4] I. Demirkol, F. Alagoz, H. Delic, and C. Ersoy, "Wireless sensor networks for intrusion detection: packet traffic modeling," IEEE Commun. Letters, vol. 10, no. 1, pp. 22-24, Jan. 2006.

[5] A. D. Wood and J. A. Stankovic, "Denial of service in sensor networks," Computer, vol. 35, no. 10, pp. 54-62, Oct. 2002.

[6] D. Liu, P. Ning, and R. Li, "Establishing pairwise keys in distributed sensor networks," ACM Transactions on Information and System Security, vol. 8, no. 1, pp. 41-77, Feb. 2005.

[7] R. A. Kemmerer and G. Vigna, "Intrusion detection: A brief history and overview," Computer, vol. 35, no. 4, pp. 27-30, Apr. 2002.

[8] J. Deng, R. Han, and S. Mishra, "Decorrelating wireless sensor network traffic to inhibit traffic analysis attacks," Pervasive and Mobile Computing, vol. 2, no. 2, pp. 159-186, Apr. 2006.

[9] J. Deng, R. Han, and S. Mishra, "Countermeasures against traffic analysis attacks in wireless sensor networks," in Proc. SecureComm 2005, Athens, Greece, pp. 113-124, 2005.

[10] P. Karn, "MACA: A new channel access method for wireless radio," in Proc. 9th Computer Networking Conference, London, ON, Canada, pp. 134-140, 1990.

[11] F. Talucci and M. Gerla, "MACA-BI (MACA by invitation) - A wireless MAC protocol for high speed ad hoc networking," in Proc. 8th IEEE International Symposium on Personal, Indoor, and Mobile Radio Communications, Helsinki, Finland, pp. 435-439, 1997.

[12] S. Singh and C. S. Raghavendra, "Power efficient MAC protocol for multihop radio networks," in Proc. 9th IEEE International Symposium on Personal, Indoor, and Mobile Radio Communications, Boston, MA, USA, pp. 435-439, 1998. 